
\title{
$\begin{array}{ll}\text { Research Square } & \begin{array}{l}\text { They should not be considered conclusive, used to inform clinical practice, } \\ \text { or referenced by the media as validated information. }\end{array}\end{array}$
}

\section{Alcohol dehydrogenase (ADH) genes family in wheat (Triticum aestivum): Genome-wide identification, characterization, phylogenetic relationship and expression patterns}

\author{
Changwei Shen \\ Henan Institute of Science and Technology \\ Jingping Yuan ( $\square$ jpyuan666@163.com ) \\ Henan Institute of Science and Technology \\ Xingqi Ou \\ Henan Institute of Science and Technology
}

\section{Research article}

Keywords: Alcohol dehydrogenase, Triticum aestivum, Genome-wide, Expression patterns, Waterlogging stress

Posted Date: August 21st, 2020

DOI: https://doi.org/10.21203/rs.3.rs-60428/v1

License: @ (i) This work is licensed under a Creative Commons Attribution 4.0 International License. Read Full License 


\section{Abstract Background}

Alcohol dehydrogenase (ADH) plays important roles in plant survival under anaerobic conditions. Although some research has been carried out the functions of $\mathrm{ADH}$ in other plants, that of wheat TaADH family genes in response to abiotic stress are unclear.

\section{Results}

A total of $22 \mathrm{ADH}$ genes were obtained from 14 chromosomes of the wheat genome by systematic screening. Multiple sequence alignment and evolutionary relationship show that these genes contain the characteristics of GroES-like domain and Zinc-binding domain, and these belong to Medium-chain -ADH type and can be divided into three subfamilies. There are 17 pairs of fragment replication genes among TaADH family members in the wheat genome, while there are 9 pairs of collinear gene pairs from ADH family members between wheat and rice genome. We speculate that these fragment repetition events may be the main reason for the amplification of TaADH family genes. Ka/Ks analysis indicated that there were 64 repetitive gene pairs, and the Ka/Ks value of these gene pairs was less than 1, which indicated that these sequences of $T a A D H$ gene were relatively conservative and did not change greatly in the process of evolution. Promoter element analysis showed that almost all of the upstream promoters of these genes contained the responsive anaerobic inducible element. Tissue localized expression and expression patterns also demonstrated that the TaADH genes responded to abiotic stress and may play an important role in waterlogging stress during the seed germination stage.

\section{Conclusions}

The results of this study may be helpful to further study the function of $T a A D H$ genes and determine the candidate gene for wheat stress resistance breeding

\section{Background}

Alcohol dehydrogenase (ADH, EC1.1.1.1) is a zinc-binding enzyme, as a dimer, dependent on NAD (P) cofactor to transform between alcohol and acetaldehyde. It was first reported that this enzyme has high activity under anoxic conditions. It mainly acts in the last step of glycolysis or fermentation under anaerobic conditions, converting acetaldehyde to alcohol [1]. ADH activity was reported for the first time from the grains and anthers of the maize [2], and the expression and activity of this gene were widespread in the "normal" state or under various pressures. The ADH gene family is actually a relatively large family, which is mainly divided into several major superfamilies, namely, short-chain dehydrogenases/reductases (SDR)-ADH (containing about 250 amino acid residues), medium-chain dehydrogenases/reductases (MDR)-ADH (containing about 350 amino acid residues) and long-chain dehydrogenases/reductases (LDR)-ADH gene superfamily (about 385-900 amino acid residues) [3,4]. The ADHs of mammals, plants and yeast belongs to MDRs, whose active sites usually contain zinc ligands. Most plant ADHs are dependent on Zn-MDRs. There are many reports about the genes of this family in recent years, and some studies mainly focus on the relationship between ADHs and aroma synthesis during fruit development [5-8], these are only part of its function, while the other functions of ADHs remain to be explained.

As $\mathrm{ADH}$ activity is considered to be a necessary condition for plant survival under anaerobic conditions, it has been the focus on the study the response of the $\mathrm{ADH}$ gene in the plant to anaerobic stress. In Arabidopsis thaliana, it was found that when the seedlings grew on the plate, the AtADH gene was constitutively expressed in the root tissue (including lateral roots), but not in the green aerial tissue, and this expression pattern was similar to the expression pattern of ADH1 in maize [9]. However, so far, there are no systematic reports on the number of ADH genes in Arabidopsis thaliana and maize genomes. Three members of the $H v A D H$ family are found in barley. When the plant is attacked by pathogens, $H v A D H-1, H v A D H-2$, and $H v A D H-3$ were induced to express under anaerobic stress, in which the expression of $H V A D H-1$ and $A D H$ activity was very high [10]. Their expression levels are not only related to oxygen stress, dehydration, low temperature, abscisic acid, and other stresses but also involved in the seedling stage, pollen, and fruit development. A total of 12 ADH genes and one formaldehyde dehydrogenease (FDH) gene were found in melon (Cucumis melo L.) genome [11]. Ten of these genes belong to the medium-chain ADH subfamily, and $\mathrm{CmFDH} 1$ belongs to the Class III $\mathrm{ADH}$, and $\mathrm{CmADH} 12$ belongs to the long-chain $\mathrm{ADH}$ subfamily. By analyzing the promoter elements of these genes, it is found that $\mathrm{CmADH}$ s is involved in the response of hormones such as ABA, IAA, and ethylene. Qin et al. [8] found eight PbrADH genes in the pear genome with intron 8-9. These Pbr-ADH proteins have many conserved domains, such as the GroES-like domain (35-164 amino acids) and the zinc-binding domain (206-340 amino acids), which belong to the typical ADH family.

Wheat is an important food crop widely planted in the world, and it is ranked first in the harvest area among the three major food crops (rice, wheat, and corn). China is the largest wheat-producing and -consuming country in the world, and what is of great significance to China's food security and farmers' increasing incomes. According to the World Food and Agriculture Organization (FAO), approximately $10 \%$ of the world's land area is affected by waterlogging to varying degrees [12]. In the Mediterranean region, the germination and growth stage of winter wheat is vulnerable to waterlogging because approximately $40 \%$ of the annual rainfall occurs during the sowing period of winter wheat [13]. The wheat-growing areas in south-central China also face the same problems [14]. For example, some areas often encounter continuous rainy days during the wheat-planting period. In addition, uneven terrain or poor drainage systems of farmland easily cause water logging or waterlogging in the soil, which often leads to a lack of oxygen supply to the soil and the inhibition of seed germination, thereby reducing the germination rate of wheat [15] and resulting in wheat reductions. Wheat seeds are under anaerobic stress after waterlogging, and $A D H$ genes will be rapidly induced and expressed. Studies on waterlogging tolerance in wheat at different stages have been reported in the early stage [16], but the expression pattern of the $A D H$ genes of wheat in response to waterlogging stress is not clear. With the release of the wheat genome, it provides a simple method for identifying new members of ADH family genes. In this study, we identified $22 \mathrm{ADH}$ family genes in wheat through sequence alignment among the wheat whole genome (IWGSC RefSeq v1.0; IWGSC2018) and Arabidopsis, rice and melon genomes, which greatly expanded the previously reported (NCBI) of 
six genes in wheat. By comparison and phylogenetic analysis with other species, it was found that these ADH family genes belong to the medium-chain ADH subgroup. In this study, the structure, promoter, tissue-specific expression, and expression of $22 \mathrm{TaADH}$ genes in responses to waterlogging stress of two wheat varieties with different sensitivity, Bainong 607 and Zhoumai 22, were investigated. The information obtained from this study will greatly promote our understanding of the gene function of the ADH family.

\section{Results}

\section{Genome-wide identification}

A total of $22 \mathrm{TaADH}$ genes in the wheat genome were further identified by BLAST with $26 \mathrm{ADH}$ genes reported in muskmelon as bait and wheat genome database using ADH domains (PF00107.26, PF08240.12, and PF13602.6) through HMMER software. According to the location distribution of these genes on chromosomes, they were named TaADH1-TaADH22 (Table 1, Table S1). Except for chromosomes 2 and 3, TaADH genes were distributed on all chromosomes, including 6 genes on chromosome 4 and one $T a A D H$ gene on chromosomes 5, 6, and 7, respectively. The number of the intron for all $22 \mathrm{TaADH}$ genes ranged from 7 to 9, while that of the exons ranged from 8 to 10. The length of amino acid in TaADH genes ranged from 347 to 415 . The range of pl was from 5.68 to 8.2 , and the molecular weight was among $34.4-44.2 \mathrm{KDa}$. Through the subcellular localization prediction of these genes, it was found that they were all located in the cytoplasm.

Table 1

Properties and locations of the predicted TaADH proteins in T. aestivum.

\begin{tabular}{|c|c|c|c|c|c|c|c|c|c|c|c|c|}
\hline \multirow[t]{2}{*}{ Gene ID } & \multirow[t]{2}{*}{ NCBI } & \multirow[t]{2}{*}{$\begin{array}{l}\text { Gene } \\
\text { name }\end{array}$} & \multirow[t]{2}{*}{ Ta_Chr } & \multirow[t]{2}{*}{ Start } & \multirow[t]{2}{*}{ End } & \multirow[t]{2}{*}{ exon } & \multirow[t]{2}{*}{ intron } & \multirow{2}{*}{$\begin{array}{l}\text { CDs } \\
\text { length } \\
\text { (bp) }\end{array}$} & \multirow{2}{*}{$\begin{array}{l}\text { Number } \\
\text { of } \\
\text { amino } \\
\text { acid }\end{array}$} & \multirow[t]{2}{*}{$\mathrm{pl}$} & $\begin{array}{l}\text { Molecular } \\
\text { weight }\end{array}$ & \multirow[t]{2}{*}{$\begin{array}{l}\text { Subc } \\
\text { local }\end{array}$} \\
\hline & & & & & & & & & & & $\mathrm{Mw} / \mathrm{KDa}$ & \\
\hline TraesCS1A02G370100.1 & & TaADH1 & $1 \mathrm{~A}$ & 547391832 & 547395833 & 9 & 8 & 1143 & 380 & 6.08 & 41.6 & Cyto \\
\hline TraesCS1A02G370200.1 & & TaADH2 & $1 \mathrm{~A}$ & 547410788 & 547417410 & 9 & 8 & 1137 & 378 & 5.87 & 40.8 & Cyto \\
\hline TraesCS1B02G389200.1 & & $T a A D H 3$ & $1 \mathrm{~B}$ & 622706402 & 622708971 & 9 & 8 & 1469 & 379 & 6.28 & 41.1 & Cyto \\
\hline TraesCS1D02G376300.1 & ADH3D & TaADH4 & 1D & 452625185 & 452627671 & 9 & 8 & 1484 & 379 & 6.03 & 41.1 & Cyto \\
\hline TraesCS4A02G202100.2 & $\mathrm{ADH} 1 \mathrm{~A}$ & TaADH5 & $4 \mathrm{~A}$ & 491715851 & 491719316 & 10 & 9 & 1140 & 379 & 6.15 & 41.0 & Cyto \\
\hline TraesCS4A02G202200.1 & $\mathrm{ADH} 2 \mathrm{~A}$ & TaADH6 & $4 \mathrm{~A}$ & 491914927 & 491917719 & 9 & 8 & 1430 & 379 & 5.81 & 40.9 & Cyto \\
\hline TraesCS4A02G202300.1 & $\mathrm{ADH} 2 \mathrm{D}$ & TaADH7 & $4 \mathrm{~A}$ & 492029965 & 492032871 & 9 & 8 & 1718 & 379 & 5.97 & 41.0 & Cyto \\
\hline TraesCS4B02G106300.1 & ADH1D & TaADH8 & $4 \mathrm{~B}$ & 115556136 & 115560148 & 10 & 9 & 1962 & 379 & 6.03 & 41.0 & Cyto \\
\hline TraesCS4B02G106400.1 & & TaADH9 & $4 \mathrm{~B}$ & 115845355 & 115848000 & 9 & 8 & 1348 & 376 & 5.91 & 40.5 & Cyto \\
\hline TraesCS4B02G106500.1 & & TaADH10 & $4 \mathrm{~B}$ & 115879177 & 115881956 & 9 & 8 & 1611 & 379 & 5.9 & 34.4 & Cyto \\
\hline TraesCS4D02G103000.1 & $\mathrm{ADH} 1 \mathrm{~A}$ & TaADH11 & $4 \mathrm{D}$ & 81918232 & 81921969 & 10 & 9 & 1839 & 379 & 6.15 & 41.0 & Cyto \\
\hline TraesCS4D02G103100.1 & & TaADH12 & $4 \mathrm{D}$ & 81971499 & 81974375 & 9 & 8 & 1467 & 379 & 5.92 & 40.9 & Cyto \\
\hline TraesCS4D02G103300.1 & & $T a A D H 13$ & $4 \mathrm{D}$ & 81984987 & 81987448 & 8 & 7 & 1044 & 347 & 6.56 & 37.6 & Cyto \\
\hline TraesCS5A02G193900.1 & & TaADH14 & $5 \mathrm{~A}$ & 397249660 & 397251898 & 9 & 8 & 1359 & 365 & 5.68 & 39.7 & Cyto \\
\hline TraesCS5B02G189200.1 & & TaADH15 & $5 \mathrm{~B}$ & 341062699 & 341068539 & 9 & 8 & 1140 & 379 & 5.83 & 40.9 & Cyto \\
\hline TraesCS5D02G196300.2 & & $T a A D H 16$ & $5 \mathrm{D}$ & 299832208 & 299835185 & 8 & 7 & 1751 & 379 & 5.68 & 41.0 & Cyto \\
\hline TraesCS6A02G386600.1 & & TaADH17 & $6 \mathrm{~A}$ & 603279456 & 603282956 & 9 & 8 & 1367 & 381 & 6.55 & 40.6 & Cyto \\
\hline TraesCS6B02G425700.1 & & TaADH18 & $6 \mathrm{~B}$ & 694401891 & 694405637 & 9 & 8 & 1529 & 381 & 6.37 & 40.7 & Cyto \\
\hline TraesCS6D02G371200.1 & & TaADH19 & $6 \mathrm{D}$ & 456554723 & 456558968 & 9 & 8 & 1629 & 381 & 6.37 & 40.7 & Cyto \\
\hline TraesCS7A02G322200.1 & & TaADH2O & $7 \mathrm{~A}$ & 466247186 & 466249779 & 8 & 7 & 1248 & 415 & 8.2 & 44.0 & Cyto \\
\hline TraesCS7B02G223100.1 & & $T a A D H 21$ & $7 \mathrm{~B}$ & 419035483 & 419038096 & 8 & 7 & 1248 & 415 & 8.18 & 44.2 & Cyto \\
\hline TraesCS7D02G319100.1 & & $T a A D H 22$ & $7 D$ & 407849007 & 407852341 & 8 & 7 & 2011 & 415 & 8.2 & 44.1 & Cyto \\
\hline
\end{tabular}

\section{Alignment and evolutionary analysis}

By comparing protein sequences of these $22 \mathrm{TaADH}$ genes, it was found that most of the residues of these protein sequences were the same. Pfam scanning of the sequences showed that all of these sequences contained the characteristic motifs of ADH (GroES-like domain and Zinc-binding domain) (Fig. 1A), in which the residues of the GroES-like domain were within 35-164 amino acid, and the amino acid residues of the Zinc-binding domain were within 206-340 amino acid. However, the location of amino acid residues of the Zinc-binding domain in TaADH2O-TaADH22 was different from those of other genes (marked with a blue box). It inferred that these genes belonged to the ADH family. To examine their evolutionary relationships in wheat and the other plant species: Arabidopsis thaliana (7), Cucumis melo (13), Cucumis sativus (12), Glycine max (3), Hordeum vulgare (1), Lycopersicon esculentum (7), Oryza sativa (1) and 
Vitis vinifera (8), a phylogenetic tree was constructed by multiple sequence alignment of 22 TaADH proteins using the adjacent linkage of (NJ) method (Fig. 1B). The predicted $A D H$ genes were classified into three groups, namely short-chain ADH, medium-chain ADH and long-chain-ADH. 22 TaADH genes in wheat belonged to medium-chain $\mathrm{ADH}$ type. According to the evolutionary relationship, these genes can be divided into 3 subfamilies: Class I contained the largest number of TaADHs (15 genes, TaADH1-9 and TaADH11-15), followed by Class II (4 genes, TaADH10 and TaADH17-19) and III (3 genes, TaADH20-22).

\section{Conservative domain analysis}

Through the conservative analysis of $T a A D H$ genes in the wheat genome, it was found that the exon of $T a A D H$ genes in Class I had 9, and the distribution of

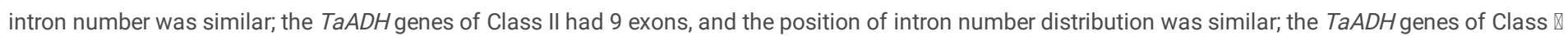
had 8 exons (Fig. 2A). In order to further clarify the protein structure of TaADHs family members in wheat, we identified the conserved motif (Fig. 2B and Fig S1) using MEME software, and found that the number of motifs in Class I TaADHs protein was 12 (such as Motif 1-7-4-9-2-8-5-11-6-10-3-14). However, the

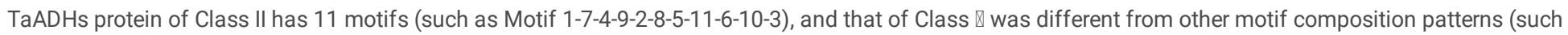
as Motif 1-4-7-12-5-13-3). In order to further analyze the functional domains of these proteins, we analyzed the functional structure of these genes (Fig. 2C). The members of wheat TaADH family have highly conserved functional domains, in which Class I TaADHs protein was mainly alcohol_DH_plant, Class II TaADHs protein was mainly alcohol_DH_class_ $\Downarrow$ domain, while Class III TaADHs protein was Zn_ADH10. Generally speaking, these TaADH family members contained the typical structural domain of alcohol_DH.

\section{Chromosomes distribution and synteny analysis}

From the distribution of TaADHs on the wheat chromosomes (Fig. 3A and 3B ), it was found that 22 TaADHs family members were mainly distributed on 15 chromosomes, of which there were 3 genes on chromosomes $4 A, 4 B$ and $4 D$, respectively. These genes have tandem replication events. To explore the collinear relationship of the TaADHs in the wheat genome and between the wheat genome and rice genome, collinear analysis was carried out by MCScanX method (Fig. 3A and 3B). We found that there were 17 fragment replication events among members of the TaADHs family in the wheat genome, including three homologous gene pairs on chromosomes 1, 6 and 7, and fragment replication in TaADH15 and TaADH16 of chromosomes 5B and 5D. However, there were 7 fragment replication events (TaADH5-TaADH8, TaADH6-TaADH9, TaADH7-TaADH10, TaADH5-TaADH11, TaADH6-TaADH12, TaADH8-TaADH11, TaADH9TaADH12) in chromosomes $4 \mathrm{~A}, 4 \mathrm{~B}$ and $4 \mathrm{D}$, which were related to the tandem replication events on chromosomes 4A, 4B and 4D. A total of 9 pairs of syntenic paralogs were found in wheat and rice genomes (Fig. 3B), in which of TaADH6 corresponds to LOC_Os11g10510.1; TaADH8 and TaADH11 correspond to LOC_Os11g10480.1; TaADH17, TaADH18, and TaADH19 correspond to LOC_Os02g57040.1; TaADH20, TaADH21, and TaADH22 correspond to LOC_Os08g01760.1.

\section{Evolutionary analysis}

The non-synonymous substitution rate (Ka), synonymous substitution rate (Ks), and Ka/Ks for 64 duplicated pairs were calculated to reveal the selection pressure of wheat TaADH family genes in the process of evolution (Fig. 4A, Table S2). It was found that the Ka/Ks of these duplicated pairs were less than 1 , which tended to a pure selection, indicating that the sequence similarity of $T a A D H$ genes was very high and relatively conservative in the process of evolution. The evolution time of the duplicated events of $\mathrm{TaADH}$ genes can be divided into three evolution periods (Fig. 4B, Table S2), of which 30 copies of TaADH duplication genes occurred about 11.19 to 16.42 million years (Mya), 12 copies of TaADH duplicated gene pairs occurred about 7.73 to 9.56 Mya, and the other 22 copies of TaADH duplicated gene pairs occurred about less than $6 \mathrm{Mya}$, the time period mostly before the wheat polyploidization event. It showed that although these genes sequences were conserved, they were different in evolutionary time.

\section{The cis-regulatory elements analysis of TaADH genes in wheat}

To further identify the cis-regulatory elements located upstream of the $T a A D H$ genes, we selected the $2 \mathrm{~K}$ bp promoter region upstream of the CDS of $T a A D H$ genes and used TBtools software to predict and visualize the cis-acting elements (Fig. 5). There were a variety of cis-acting elements in the upstream promoters of these genes, which are responsive to 11 kinds of stress (hormone response, anaerobic response, defense, and stress response, drought induction, light response, low-temperature response, etc.). Except for $T a A D H 13$, the upstream promoters of other genes contain elements (ARE), that respond to anaerobic induction, and $\mathrm{TaADH} 6$ and $\mathrm{TaADH} 9$ contained as many as six cis-regulatory elements of ARE. We also found that the upstream promoter of $T a A D H 4$ contains 8 cis-regulatory elements (ABRE) responsive to abscisic acid. The upstream promoter of TaADH3 contains as many as 14 cis-regulatory elements (TGACG-motif and CGTCA-motif) responsive to Me-jasmonic acid.

\section{Tissue-specific expression patterns of TaADH genes in different tissues and organs}

In RNA-seq data of different tissues and organs in T. aestivum from, FPKM values of transcript accumulation of 22 TaADH genes were obtained from publicly available expression data sets, and then the corresponding heatmaps of relative expression levels were generated using Heatmap tool. The transcription levels in various $T$. aestivum tissues, including the roots, leaf, stem, spike, grain, and seeding were examined (Fig. 6). We found that except for TaADH2, there was no expression of $T a A D H 6-7$ in leaves, but the expression of $T a A D H 6$ was the highest in grain and that of $T a A D H 7$ was the highest in stems. The expression pattern of $T a A D H 4$ was similar to that of $T a A D H 6$, and the expression level was the highest in grains. The TaADH1 and TaADH9 expressions were only detected in grains and roots of wheat, but not in other parts of wheat, while the expression pattern of TaADH15 was opposite to that of TaADH1 and TaADH9. Other genes ( $T a A D H 3, T a A D H 5, T a A D H 8, T a A D H 10-11, T a A D H 14, T a A D H 16-22)$ were expressed in all parts of wheat. Among them, the expression of TaADH5, $T a A D H 8, T a A D H 11$, and $T a A D H 17-22$ was the highest in wheat grains.

\section{The expression of TaADH genes in wheat seed under waterlogging treatment}


To further analyze the response of two wheat seed with different waterlogging tolerance to waterlogging stress during the germination stage, we analyzed the relative expression levels of 22 members of wheat TaADH family (Fig. 7). The results showed that the expression levels of seven TaADH genes (TaADH1/2, $T a A D H 13, T a A D H 17, T a A D H 18, T a A D H 19, T a A D H 20)$ in the seeds of the intolerant variety Zhoumai 22 were significantly up-regulated at 24 hours after waterlogging treatment compared with the control treatment, but there was no significant difference in the expression levels of $T a A D H 1 / 2, T a A D H 17$, $T a A D H 18, T a A D H 19$, and $T a A D H 2 O$ genes compared with the control treatment 72 hours after germination, only the expression level of $T a A D H 13$ gene showed an upward trend. Compared with the control treatment, the expression levels of 14 genes (TaADH1/2, TaADH3-6, TaADH8-13, TaADH19, and TaADH20) in the seeds of Bainong 607 were significantly up-regulated at 24 hours after waterlogging treatment, while the expression levels of $T a A D H 1 / 2, T a A D H 3$, and $T a A D H 9$ genes were significantly up-regulated at 72 hours after germination compared with the control treatment, while the expression levels of $T a A D H 5$, $T a A D H 6, T a A D H 14$, and $T a A D H 16$ genes decreased. The results showed that the difference between waterlogging-tolerant and non-waterlogging-tolerant varieties after waterlogging treatment was closely related to the early and rapid expression of TaADHgenes.

\section{Discussion}

In recent years, with global warming, extreme weather occurs more frequently, in which flood disaster is one of the abiotic stresses faced by plants, and hypoxia will first occur in the flooded environment. Waterlogging-tolerant plants can often stimulate alcohol fermentation and reduce lactic acid fermentation to prevent acidifying cytoplasm. To avoid cytoplasmic acidosis, ethanol production is necessary for plants to survive under anaerobic conditions [17]. The role of alcohol dehydrogenase has been reported in many species [18-20]. With the development of sequencing technology, the genome sequences of many species can be analyzed, which promotes the identification of plant gene families at the genome-wide level. The $A D H$ genes have been detected in various plants, including tomato [5], rice [21], barley [22], melon [7], and pear [8]. With the publication of the wheat genome, it is possible to systematically study the function of wheat TaADH family members.

\section{Structural characterization of TaADHs}

A total of 22 TaADH genes were identified in the wheat genome database, including 6 TaADH genes published by NCBI. In this study, we found that there are 8-10 exons and 7-9 introns of TaADH family members in wheat, which is consistent with the 8-9 introns of ADH genes reported by Strommer [23]. For example, the ADH2/3 in barley and the ADH in wheat contain 8 introns. While ADH1 and ADH3 in Arabidopsis thaliana contain 6 and 4 introns, respectively, and $\mathrm{ADH}$ in Chinese cabbage contains 5 introns [23]. The increase in the number of introns in common wheat (Triticum aestivum L.) indicated that the $T a A D H s$ family was expanding significantly in the evolution from lower plants to higher plants, and the expansion of the number of ADH members could enhance the ability of organisms to adapt to more complex environmental changes.

The plant $A D H$ family usually divides into short-chain-ADH and medium-chain-ADH. Kitaoka et al. [21] found that there was only one short-chain alcohol dehydrogenase / reductase SDRs (OSMAS/SDR110C-MS1) in rice, which belonged to the short-chain-ADH type, while the other long-chain-ADHs were rarely reported $[24,25]$. $22 \mathrm{TaADH}$ family members identified in this study belonged to medium-chain-ADH, and these genes have a highly conserved functional domain (GroES-like domain and zinc-binding domain) (Fig. 1A). This was similar to the structure of pear PbrADHs reported by Qin et al. [8]. ADH is a member of the medium-length dehydrogenase/reductase (MDR) protein superfamily. There is a typical domain of GroES-like in MDR, which is determined by chaperonin-10, based on its similarity to GroES molecular chaperone [26]. Association of Zn co-factors with a primitive MDR may have occurred in the early days of atmospheric oxygen, when $\mathrm{Zn}$ is likely to have been a preferred co-factor due to its valence stability [27]. From the perspective of evolution, $\mathrm{Vv}$-ADH2 in grape [28], $\mathrm{CmADH} 1$ in melon [29] and At-ADH1 in Arabidopsis thaliana [30] all clustered in a class of medium-chain zinc-bound ADHs (Fig. 1B). Therefore, 22 $T a A D H$ genes in wheat belonged to the typical ADHs family.

\section{Phylogenetic analysis and evolution of TaADHs}

Gene replication is an important evolutionary process of gene family expansion, and the replicated genetic material provides an opportunity for functional differentiation. Functional differences caused by gene duplication are considered to be an important factor in species formation and environmental adaptability [31]. Therefore, gene replication analysis can help us better understand the evolution of genes and species. Two repetitive genes located on the same chromosome are called tandem replication. They exist on different chromosomes but come from the same subgenome, which is called fragment replication [32]. There are 4 gene tandem replication and 17 fragment replication events among members of the TaADHs family in the wheat genome (Fig. 3A). Most of the fragment replication events occurred in homologous chromosomes, such as chromosomes 1, 4, 6, and 7. In addition, there were gene collinearity between chromosomes 4, 6 and 7 in the wheat genome and chromosomes 2, 8 and 11 in the rice genome, which indicated that wheat polyploidy was the main reason for the expansion of TaADH family members, which also confirmed the conservatism of TaADH family members on individual chromosomes.

$\mathrm{Ka}, \mathrm{Ks}$, and $\mathrm{Ka} / \mathrm{Ks}$ can well explain the history of a gene or gene region facing selection pressure [33]. In this study, we found that there were 64 duplicate gene pairs in the TaADHs family, and their Ka/ KS values were all less than 1 (Fig. 4B), which means that all duplicate gene pairs are purified and selected. The Ks value was used to estimate the occurrence time of repetitive events. The results showed that 64 repetitive events of TaADH family genes occurred between 1.55 and $16.42 \mathrm{Mya}$, including 30 repetitive gene pairs with evolution time of 11.19-16.42 Mya and 12 repetitive gene pairs with evolution time of 7.73-9.56 Mya, all of which were earlier than the first time of genomic replication in wheat [34]. The second time is that the subgenome of common wheat can be traced back to 300000 years ago, including Triticum urartu (A sub-genome) and the hybridization of the Aegilops speltoides to form tetraploid wheat ( $T$. dicoccoides, A and B sub-genome). About 8, 000 years ago, tetraploid wheat, also known as the Aegilopstauschii (D subgenome, produced hexaploid wheat ( $T$. aestivum, A, $\mathrm{B}$, and $\mathrm{D}$ subgenomes) [35]. It showed that most of the TaADH family genes in wheat have undergone strict purification and screening in the process of evolution, and retained the original function through fragment replication events.

Expression profile analysis of TaADHs

Page 5/14 
Gene promoters are important factors in regulating gene expression patterns. They regulate gene expression at the transcriptional and post-transcriptional levels [36]. Cis-acting elements in a specific promoter region participate in tissue-specific expression patterns under various environmental conditions. In the corresponding upstream region for a gene, there was a positive correlation between the number of cis-acting elements and multiple stimuli [37]. Through the prediction of cis-acting elements of wheat TaADH family members, it was found that these genes were selected by a variety of biotic and abiotic factors (Fig. 5). Alcohol dehydrogenases catalyze the reversible conversion of aldehydes to the corresponding alcohols. They are involved in the stress response of plants and are mainly responsible for the production of ethanol in an anaerobic environment. At the same time, it is also widely involved in other stress, activator, and abscisic acid responses [38]. ADH has a good protective effect on hypoxia stress after flooding [39], as well as seed development and pollen aerobic metabolism [40]. There are many isozyme genes of $A D H$ in the seed. Studies on tracking the activity of ADH during seed development have proved that $A D H$ isozyme genes are active at different times. When seeds were under hypoxia stress at the germination stage, $A D H$ activity might play a very important role in germination [23]. Three ADH genes ( $H V A D H-1, H v A D H-2$, and $H V A D H-3)$ in barley were found, in which the activity of HvADH-1 during aerobic growth could be detected, while the expression of $H V A D H-1$ and $H V A D H-2$ could be induced by hypoxia, while the expression level of $H v A D H-3$ under hypoxia was significantly lower than that of the other two genes [41]. Proels et al. [42]found that $H V A D H-1$ was the only constitutively expressed in barley seedlings. However, ADH-3 activity could not be detected in barley leaves under any conditions.

In this study, it was also found that $T a A D H 3, T a A D H 5, T a A D H 8, T a A D H 10-11, T a A D H 14$, and TaADH16-22 were expressed in all parts of wheat. This suggested that these genes might also have constitutive expression. Manangkil et al. [22]confirmed that the expression of $A D H 1$ and $A L D H 2 a$ in rice seedlings increased rapidly under submergence stress, but decreased rapidly when submergence was removed, indicating that higher express levels of $A H D 1$ and $A L D H 2 a$ might be one of the reasons why rice is more tolerant to submergence than other plants. When wheat seeds were exposed to anaerobic environment (waterlogging treatment), TaADHs was expressed differently with different exposure time. No matter the waterlogging tolerant variety Bainong 607 or the intolerant variety Zhoumai 22, the expression levels of $T a A D H 1 / 2, T a A D H 13, T a A D H 19$, and $T a A D H 20$ genes at 24 hours after waterlogging treatment were significantly higher than those of the control treatment (Fig. 7). From the tissue expression analysis, it was found that the relative expression of these genes was the highest in grains, especially the specific expression of $T a A D H 1$ and $T a A D H 13$ in wheat grains. This suggested that these genes might be induced the rapid expression under anaerobic stress. However, after 72 hours of germination, the relative expression levels of $T a A D H 1 / 2, T a A D H 3$ and TaADH9 genes in Bainong 607 seeds were significantly up-regulated compared with the control treatment. However, only the expression level of the TaADH13 gene increased at 72 hours after germination of Zhoumai 22 seed (Fig. 7). This showed that $T a A D H 1 / 2, T a A D H 3$, and $T a A D H 9$ played an important role in responding to waterlogging stress and served as an important basis for screening waterlogging-tolerant wheat varieties.

\section{Conclusions}

A total of $22 \mathrm{TaADH}$ genes were identified and analyzed at the wheat genome. These genes were distributed on 15 chromosomes, of which there were 3 homologous genes on chromosomes $4 \mathrm{~A}, 4 \mathrm{~B}$, and $4 \mathrm{D}$, respectively, and there were tandem replication events in these genes. According to Pfam domain compositions, it was found that all these protein sequences contained the characteristic motifs of ADH (GroES-like domain and Zinc-binding domain). Through phylogenetic tree analysis with other species, it was found that $22 \mathrm{TaADH}$ genes in wheat belonged to medium-chain $\mathrm{ADH}$ type and were grouped into 3 subfamilies. Collinearity analysis within the wheat genome and between the wheat genome and rice genome showed that there were 17 fragment replication events among members of the TaADH family, while the number of collinear ADH gene pairs of wheat and rice was 9 pairs. According to the Ka/Ks and evolutionary years, it was found that there were 64 repetitive gene pairs with $\mathrm{Ka} / \mathrm{Ks}$ less than 1 , which tended to purification selection, indicating that strong purifying selection had taken place in the process of evolution of TaADH genes. Cis-acting elements and tissue expression analysis showed that these genes were responsive to 11 kinds of abiotic stress. TaADH3, TaADH5, TaADH8, TaADH10-11, TaADH14, and TaADH16-22 were expressed in all parts of wheat, belonging to constitutive expression, and other genes were specifically expressed. By comparing the expression profiles of waterlogging-tolerant wheat Bainong 607 and waterlogging-intolerant wheat Zhoumai 22 at different germination stages after waterlogging treatment, it was found that TaADH1/2, $\mathrm{TaADH} 3$ and $T a A D H 9$ played an important role in responding to waterlogging stress, and could be used as an important basis for screening waterloggingtolerant wheat varieties. These results will provide valuable information regarding further functional elucidation of $T a A D H$ genes in wheat.

\section{Methods}

\section{Wide-genome identification of $T a A D H$ genes in wheat}

The whole-genome data was downloaded from the wheat genome database

(https://urgi.versailles.inra.fr/download/iwgsc/IWGSC_RefSeq_Assemblies/v1.0/) [43] and was used to identify ADHs candidate genes in wheat with two methods. First of all, $26 \mathrm{ADH}$ genes reported in melon were used as the inquiry to search for the homologous gene of $A D H s$ through the BLAST function of BLAST2.3.0 + tool provided by the National Center for Biotechnology Information (NCBI). ADH genes of melon were downloaded from the reported melon genome database. Secondly, we download the ADH domains (PF00107.26, PF08240.12, and PF13602.6) from the PFAM website (http://pfam.xfam.org/) to further identify the domains of these candidate genes using HMMER software. Finally, ExPASy (http://prosite.expasy.org/)) was used to verify the integrity of the ADH proteins domain, and motif analysis was performed for ADH proteins using the Batch-CDD. The maximum number of motifs was set to 15 . Each $\mathrm{ADHs}$ gene is renamed according to its exact location on the chromosome (from top to bottom each chromosome). The protein molecular weight and isoelectric point of the candidate genes were calculated by ExPASy website (https://web.expasy.org/compute_pi/). Plant-mPLoc (http://www.csbio.sjtu.edu.cn/bioinf/plant-multi) was used to predict the subcellular location of the TaADH genes [44].

\section{Sequences alignment and evolutionary analysis of TaADH genes in wheat}

The ADH protein sequences were downloaded from the genomes of Arabidopsis thaliana, Chinese spring wheat, rice, and muskmelon respectively. The protein sequences of these species were compared by ClustalW2 (http://www.genome.jp/tools-bin/CLUSTORW) software, and the phylogenetic tree was constructed

Page 6/14 
by the Neighbor-Joing (NJ) method in MEGA7.0 (Test of phylogeny: Bootstrap method, Bootstrap replications: 1000) [45].

\section{Gene structure and motif distribution of $T a A D H$ genes in wheat}

The introns and exons of the ADH genes were generated by using the Gene Structure Display Server 2. 0 (GSDS2.0, http://gsds.cbi.pku.edu.cn/). The protein sequence of $A D H s$ was analyzed by online software Multiple EM for Motif Elicitation (MEME, http://meme-suite.org/tools/meme [46]. The number of motifs is set to 15. Visualization of the results were performed by the TBtools (https://github.com/CJ-Chen/TBtools) [47].

\section{Gene duplications event and $\mathrm{Ka} / \mathrm{Ks}$ of $\mathrm{TaADH}$ genes in wheat}

In order to identify gene duplications event, all CDS sequences of wheat $A D H$ genes were subjected to BLAST searches against each other (identity $>80 \%$, e value $<1 \mathrm{e}^{-10}$ ) by using the local Blast program. Gene alignment coverage was then acquired by pair-wise alignment using the previously calculated method: Gene alignment coverage = (alignment length-mismatch length) / the length of larger genes. When the gene alignment coverage is more than 0.75 , it is considered to be a duplicate gene pair. In addition, in the $100 \mathrm{~kb}$ region, two genes separated by five or fewer genes are considered as tandemly duplicated genes, while replication genes between different chromosomes are defined as fragment repeat genes. The values of Ka/ Ks between Ka and Ks and between paired genes were calculated by DnaSP software (http://www.ub.edu/dnasp/). For the timing of duplication events, the formula: $\mathrm{T}=\mathrm{Ks} / 2 \lambda \times 10^{-6} \mathrm{Mya}$ was used to calculate divergence time $(T)$ in millions of years (Mya), where $\lambda=6.5 \times 10^{-9}$ represented the rate of replacement of each locus per herb plant year [48].

\section{Chromosome mapping and collinearity analysis of TaADH genes in wheat}

The chromosome locations of the $T a A D H$ genes from the Chinese spring wheat genome

(https://urgi.versailles.inra.fr/download/iwgsc/IWGSC_RefSeq_Assemblies/v1.0/) were determined using the TBtools [47]. To confirm gene homology, the protein sequences of $T a A D H$ genes in wheat were blasted against each other by BLASTP (E value $<10^{-20}$, identity $>75 \%$ ). The chromosome distribution and collinearity of these $A D H s$ genes were visualized by Circos [49].

\section{Promoter cis-acting element analysis of $\mathrm{TaADH}$ genes in wheat}

To analyze the promoter elements of $T a A D H$ genes, the promoter sequences (2000 bp before the start codon) of all TaADH genes were extracted from the Chinese spring wheat database. The cis-acting elements of these genes were predicted by online tool PLANTCARE

(https://bioinformatics.psb.ugent.be/webtools/plantcare/html/) [50] and visualized by TBtools (https://github.com/CJ-Chen/TBtools) [47].

\section{Expression pattern of $\mathrm{TaADH}$ genes in wheat}

To analyze the expression profiles of $T a A D H$ genes in wheat, RNA-Seq data (http://www.wheat-expression.com/) of different parts of Chinese spring wheat from the reported database were downloaded, and the FPKM data of all TaADH genes were extracted [51]. Heatmap (https://github.com/CJ-Chen/TBtools) were drawn with TBtools software [47].

\section{Analysis of expression of $\mathrm{TaADH}$ genes under waterlogging stress}

Experimental treatment: waterlogging treatment was $72 \mathrm{~h}$ at $20^{\circ} \mathrm{C}$ constant temperatures in the dark for fifty plump seed placed in the glass Petri dishes. At the end of waterlogging treatment, the seeds were transferred and germinated in a Petri dish containing one layer filter paper with $10 \mathrm{~mL}$ of sterilized deionized water. Control treatment: a control (CK), consisting of not water-logged seed, was added for each variety. Germination was conducted on the seed as same as waterlogging treatment. The experimental and control treatments were given 5 repetitions, each containing 50 seeds. All seeds were grown in a growth chamber at $25^{\circ} \mathrm{C}$ and $75 \%$ relative humidity ( $16 \mathrm{~h}$ light/ $8 \mathrm{~h}$ dark cycles). In this study, two wheat (Triticum aestivum L.) varieties, 'Zhoumai 22 ' (ZM22, waterlogging-intolerant) and 'Bainong 607' (BN607, waterlogging-tolerant), were selected as the materials. ZM22 is a commercial winter wheat cultivar in Henan Province, China. The BN207 and BN607 seeds used in this study were cultivated by Prof. Xingqi Ou from the School of Life Science and Technology, Henan Institute of Science and Technology, Xinxiang, China. All the seeds were provided by the School of Life Science and Technology, Henan Institute of Science and Technology.

To explore the response of wheat seeds to waterlogging stress, samples of embryo and endosperm of germinated seeds from ZM22 and BN607 were collected at $24 \mathrm{~h}$ and $72 \mathrm{~h}$ of germination under the waterlogging and control treatments. Total RNA was removed with RNase-free DNase I (Takara, Tokyo, Japan) to avoid genomic DNA contamination. First-strand cDNAs were synthesized using the PrimeScript RT Reagent Kit with gDNA Eraser (Takara, Dalian, China) according to the manufacturer's protocol. The qRT-PCR assays were performed with the Primer Script RT Reagent Kit (Takara, Dalian, China). The 18S (AJ272181.1) was used as a reference gene. Data were analyzed with Opticon monitor software (Bio-Rad). All primers for qRT-PCR were designed using Primer 6.0 software and primer sequences are listed in Table S3. The PCR conditions were as follows: $95^{\circ} \mathrm{C}$ for $10 \mathrm{~s}$ and $40 \mathrm{cycles}$ of $95{ }^{\circ} \mathrm{C}$ for $5 \mathrm{~s}$ and $60{ }^{\circ} \mathrm{C}$ for $30 \mathrm{~s}$. The qRT-PCRs were performed using an ABI Step One Plus. All the experiments were performed with three biological replicates. One sample constitutes a mixture of three seeds. The relative expression was calculated using the $2^{-\Delta \Delta C t}$ method [52]

\section{Abbreviations}

ZM22: Zhoumai 22; BN607:Bainong 607; ADH:Alcohol Dehydrogenase; mRNA:Messenger RNA; qRT-PCR:Quantitative Real-time PCR; FPKM:Fragments Per Kilobase Per Million Mapped Reads; NCBI:National Center for Biotechnology Information; MW:Molecular weight; pl:Isoelectric points; Ka:Non-synonymous substitution rate, Ks:Synonymous substitution rate; SDR-ADH:Short-chain dehydrogenases/reductases; MDR-ADH:Medium-chain dehydrogenases/reductases; LDR-ADH:Long-chain dehydrogenases/reductases; FDH:Formaldehyde dehydrogenease.

\section{Declarations}




\section{Availability of data and materials}

All data generated or analyzed during this study were included in this published article and the additional files.

\section{Consent for publication}

Not applicable

\section{Competing interests}

No potential conflict of interest was reported by the authors.

\section{Ethics approval and consent to participate}

Two wheat cultivars, 'Zhoumai 22' (ZM22) and 'Bainong 607' (BN607), were used in the present study. ZM22 originated from a cross of three breeding lines, namely Zhoumai 12, Wenmai 6 and Zhoumai 13. The BN607 seeds used in this study were cultivated by Prof. Xingqi Ou from the School of Life Science and Technology, Henan Institute of Science and Technology, Xinxiang, China.

\section{Consent for publication}

Not applicable.

\section{Competing interests}

The authors declare that they have no competing interests.

\section{Funding}

This research was financially supported by the Science and Technology Program of Henan Province (202102110159) and the Scientific Research Foundation for High - level Talent (2017034) to C.S., the Scientific Research Foundation for High - level Talent (103010620001/015 and 2017034) to J.Y., the Major Science and Technology Project in Xinxiang (ZD18007) to X.O.. The funding bodies played no role in the design of the study or collection, analysis and interpretation of data and in writing this manuscript.

\section{Acknowledgements}

We are grateful to Prof. Xingqi Ou from the School of Life Science and Technology, Henan Institute of Science and Technology for the seeds.

\section{References}

1. Hageman RH, Flesher D. The effect of an anaerobic environment on the activity of alcohol dehydrogenase and other enzymes of corn seedlings. Arch Biochem Biophys. 1960;87(2):203-9.

2. Schwartz D. Genetic Control of Alcohol Dehydrogenase-a Competition Model for Regulation of Gene Action. Genetics. 1971;67(3):411 - 25.

3. Alka K, Windle HJ, Cornally D, Ryan BJ, Henehan GTM. A short chain NAD(H)-dependent alcohol dehydrogenase (HpSCADH) from Helicobacter pylori: A role in growth under neutral and acidic conditions. Int J Biochem Cell B. 2013;45(7):1347-55.

4. Jornvall H, Hedlund J, Bergman T, Kallberg Y, Cederlund E, Persson B. Origin and evolution of medium chain alcohol dehydrogenases. Chem-Biol Interact. 2013;202(1):91-6.

5. Moummou H, Tonfack LB, Chervin C, Benichou M, Youmbi E, Ginies C, Latche A, Pech J. Functional characterization of SlscADH1, a fruit-ripeningassociated short-chain alcohol dehydrogenase of tomato. J Plant Physiol. 2012;169(15):1435-44., Der Rest BV.

6. Xu XW, Wang HH, Qi XH, Xu Q, Chen XH. Waterlogging-induced increase in fermentation and related gene expression in the root of cucumber (Cucumis sativus L.). Sci Hortic. 2014;179:388-95.

7. Jin YZ, Zhang C, Liu W, Tang YF, Qi HY, Chen H, Cao SX. The alcohol dehydrogenasegene family in melon (Cucumis melo L.): bioinformatic analysis and expression patterns. Front Plant Sci. 2016;7(26).

8. Qin G, Xiaoxiao QI, Yongjie Q, Gao Z, Xingkai Y, Pan H, Yiliu X. Identification and expression patterns of alcohol dehydrogenase genes involving in ester volatile biosynthesis in pear fruit. J Integr Agr. 2017;16(8):1742-50.

9. Freeling M, Bennett DC. Maize Adh1. Annual Review Genetics. 1984;19:297-323.

10. Good AG, Crosby WL. Induction of alcohol dehydrogenase and lactate dehydrogenase in hypoxically induced barley. Plant Physiol. 1989;90(3):860-6.

11. Jin Y, Zhang C, Liu W, Qi H, Chen H, Cao S. The cinnamyl alcohol dehydrogenase gene family in melon (Cucumis melo L.): bioinformatic analysis and expression patterns. PLOS ONE. 2014;9(7):670.

12. Li C, Jiang D, Wollenweber B, Li Y, Dai T, Cao W. Waterlogging pretreatment during vegetative growth improves tolerance to waterlogging after anthesis in wheat. Plant Sci. 2011;180(5):672 - 78. 
13. Bassu S, Asseng S, Motzo R, Giunta F. Optimising sowing date of durum wheat in a variable Mediterranean environment. Field Crop Res. 2009;111(1):109 $-18$.

14. Arduini I, Orlandi C, Ercoli L, Masoni A. Submergence sensitivity of durum wheat, bread wheat and barley at the germination stage. Ital J Agron. 2016;11(2):100-6.

15. Kırmızı S, Bell RW. Responses of barley to hypoxia and salinity during seed germination, nutrient uptake, and early plant growth in solution culture. J Plant Nutr Soil Sc. 2012;175(4):630 - 40.

16. Herzog M, Striker GG, Colmer TD, Pedersen O. Mechanisms of waterlogging tolerance in wheat - a review of root and shoot physiology. Plant Cell Environ. 2016;39(5):1068-86.

17. Roberts JKM, Callis J, Jardetzky O, Walbot V, Freeling M. Cytoplasmic acidosis as a determinant of flooding intolerance in plants. Proc Natl Acad Sci USA. 1984;81(19):6029-33.

18. Sasnauskas K, Jomantien R, Januska A, Lebedien E, Lebedys J, Janulaitis A. Cloning and analysis of a Candida maltosa gene which confers resistance to formaldehyde in Saccharomyces cerevisiae. Gene. 1992;122(1):207 - 11.

19. Gutheil WG, Holmquist B, Vallee BL. Purification, characterization, and partial sequence of the glutathione-dependent formaldehyde dehydrogenase from Escherichia coli: a class III alcohol dehydrogenase. Biochemistry. 1992;31(2):475 - 81.

20. Hjelmqvist L, Shafqat J, Siddiqi AR, Jornvall H. Alcohol dehydrogenase of class III: consistent patterns of structural and functional conservation in relation to class I and other proteins. FEBS Lett. 1995;373(3):212-6.

21. Kitaoka N, Wu Y, Zi J, Peters RJ. Investigating inducible short-chain alcohol dehydrogenases/reductases clarifies rice oryzalexin biosynthesis. Plant $\mathrm{J}$. 2016;88(2):271-9.

22. Manangkil OE, Rafael AB, Nakamura C. Gene expression profiling in rice (Oryza sativa L.) cultivars with different levels of seedling vigor under submergence stress. Philippine J Crop Sci. 2019;44(2):1-12.

23. Strommer J. The plant ADH gene family. Plant J. 2011;66(1):128 - 42.

24. Thompson CE, Salzano FM, De Souza ON, Freitas LB. Sequence and structural aspects of the functional diversification of plant alcohol dehydrogenases. Gene. 2007;396(1):108 - 15.

25. Borras E, Albalat R, Duester G, Pares X, Farres J. The Xenopus alcohol dehydrogenase gene family: characterization and comparative analysis incorporating amphibian and reptilian genomes. BMC Genomics. 2014;15(1):216.

26. Taneja B, Mande S. Conserved structural features and sequence pattern in the GroES fold family. Protein Eng. 1999;12.

27. Jörnvall H, Hedlund J, Bergman T, Oppermann U, Persson B. Superfamilies SDR and MDR: From early ancestry to present forms. Emergence of three lines, a Zn-metalloenzyme, and distinct variabilities. Biochem Bioph Res Co. 2010;396(1):125 - 30.

28. Tesnière $C$, Verriès $C$. Molecular cloning and expression of CDNAs encoding alcohol dehydrogenases from Vitis vinifera L. during berry development. Plant Sci. 2000;157(1):77-88.

29. Manríquez D, El-Sharkawy I, Flores FB, El-Yahyaoui F, Regad F, Bouzayen M, Latché A, Pech J-C. Two highly divergent alcohol dehydrogenases of melon exhibit fruit ripening-specific expression and distinct biochemical characteristics. Plant Mol Biol. 2006;61(4):675.

30. Cheng F, Hu T, An Y, Huang J, Xu Y. Purification and enzymatic characterization of alcohol dehydrogenase from Arabidopsis thaliana. Protein Expres Purif. 2013;90(2):74 - 7.

31. Salse J, Bolot S, Throude M, Jouffe V, Piegu B, Quraishi UM, Calcagno T, Cooke R, Delseny M, Feuillet C. Identification and Characterization of Shared Duplications between Rice and Wheat Provide New Insight into Grass Genome Evolution. Plant Cell. 2008;20(1):11-24.

32. He H, Dong Q, Shao Y, Jiang H, Zhu S, Cheng B, Xiang Y. Genome-wide survey and characterization of the WRKY gene family in Populus trichocarpa. Plant Cell Rep. 2012;31(7):1199 - 217.

33. Hurst LD. The Ka/Ks ratio: diagnosing the form of sequence evolution. Trends Genet. 2002;18(9):486-7.

34. Ling H, Zhao S, Liu D, Wang J, Sun H, Zhang C, Fan H, Li D, Dong L, Tao Y. Draft genome of the wheat A-genome progenitor Triticum urartu. Nature. 2013;496(7443):87-90.

35. Fu J, Yu H, Li X, Xiao J, Wang S. Rice GH3 gene family: Regulators of growth and development. Plant Signal Behavr. 2011;6(4):570-4.

36. Higo K, Ugawa Y, Iwamoto M, Higo H. PLACE: a database of plant cis-acting regulatory DNA elements. Nucleic Acids Res. 1998;26(1):358-9.

37. Tran L-S, Nakashima K, Sakuma Y, Osakabe Y, Qin F, Simpson S, Maruyama K, Fujita Y, Shinozaki K, Yamaguchi-Shinozaki K. Co-expression of the stressinducible zinc finger homeodomain ZFHD1 and NAC transcription factors enhances expression of the ERD1 gene in Arabidopsis. Plant J. 2007;49:46-63.

38. Peters JS, Frenkel C. Relationship between alcohol dehydrogenase activity and low-temperature in two maize genotypes, Silverado F1 and Adh1-Adh2doubly null. Plant Physiol Bioch. 2004;42(10):841-6.

39. Bailey-Serres J, Voesenek LACJ. Flooding Stress: Acclimations and Genetic Diversity. Annual Annual Rev Plant Biol. 2008;59(1):313 - 39.

40. Macnicol PK, Jacobsen JV. Regulation of alcohol dehydrogenase gene expression in barley aleurone by gibberellin and abscisic acid. Physiol Plant. 2001;111(4):533-9.

41. Kasbauer CL, Pathuri IP, Hensel G, Kumlehn J, Huckelhoven R, Proels RK. Barley ADH-1 modulates susceptibility to Bgh and is involved in chitin-induced systemic resistance. Plant Physiol Biochem. 2018;123:281-7.

42. Proels RK, Westermeier W, Hückelhoven R. Infection of barley with the parasitic fungus Blumeria graminis f.sp. hordei results in the induction of HvADH1 and HvADH2. Plant Signal Behav. 2011;6(10):1584-7. 
43. Appels R, Eversole K, Stein N, Feuillet C, Keller B, Rogers J, Pozniak CJ, Choulet F, Distelfeld A, Poland J. Shifting the limits in wheat research and breeding using a fully annotated reference genome. Science. 2018;361(6403).

44. Fang Z, Jiang W, He Y, Ma D, Liu Y, Wang S, Zhang Y, Yin J. Genome-Wide Identification, Structure Characterization, and Expression Profiling of Dof Transcription Factor Gene Family in Wheat (Triticum aestivum L.). Agronomy. 2020;10(2):294.

45. Zhu Y, Yang L, Liu N, Yang J, Zhou X, Xia Y, He Y, He Y, Gong H, Ma D. Genome-wide identification, structure characterization, and expression pattern profiling of aquaporin gene family in cucumber. BMC Plant Biol. 2019;19(1):345.

46. Bailey TL, Williams N, Misleh C, Li WW. MEME: discovering and analyzing DNA and protein sequence motifs. Nucleic Acids Res. 2006;34:369 - 73.

47. Chen C, Chen H, Zhang Y, Thomas HR, Frank MH, He Y, Xia R. TBtools: an integrative toolkit developed for interactive analyses of big biological data. Mol Plant. 2020;13(8):1194 - 202.

48. Rozas J, Sanchezdelbarrio JC, Messeguer X, Rozas R. DnaSP. DNA polymorphism analyses by the coalescent and other methods. Bioinformatics. 2003;19(18):2496-7.

49. Krzywinski M, Schein JE, Birol I, Connors JM, Gascoyne RD, Horsman D, Jones SJM, Marra MA. Circos: An information aesthetic for comparative genomics. Genome Res. 2009;19(9):1639-45.

50. Lescot M, Dehais P, Thijs G, Marchal K, Moreau Y. De Peer YV, Rouze P, Rombauts S. PlantCARE, a database of plant cis-acting regulatory elements and a portal to tools for in silico analysis of promoter sequences. Nucleic Acids Res. 2002;30(1):325-7.

51. Borrill P, Ramirez-Gonzalez R, Uauy C. expVIP: a Customizable RNA-seq Data Analysis and Visualization Platform. Plant Physiol. 2016;170(4):2172-86.

52. Livak KJ, Schmittgen TD. Analysis of relative gene expression data using real-time quantitative PCR and the 2(-Delta Delta C(T)) Method. Methods. 2001;25(4):402-8.

\section{Figures}

A
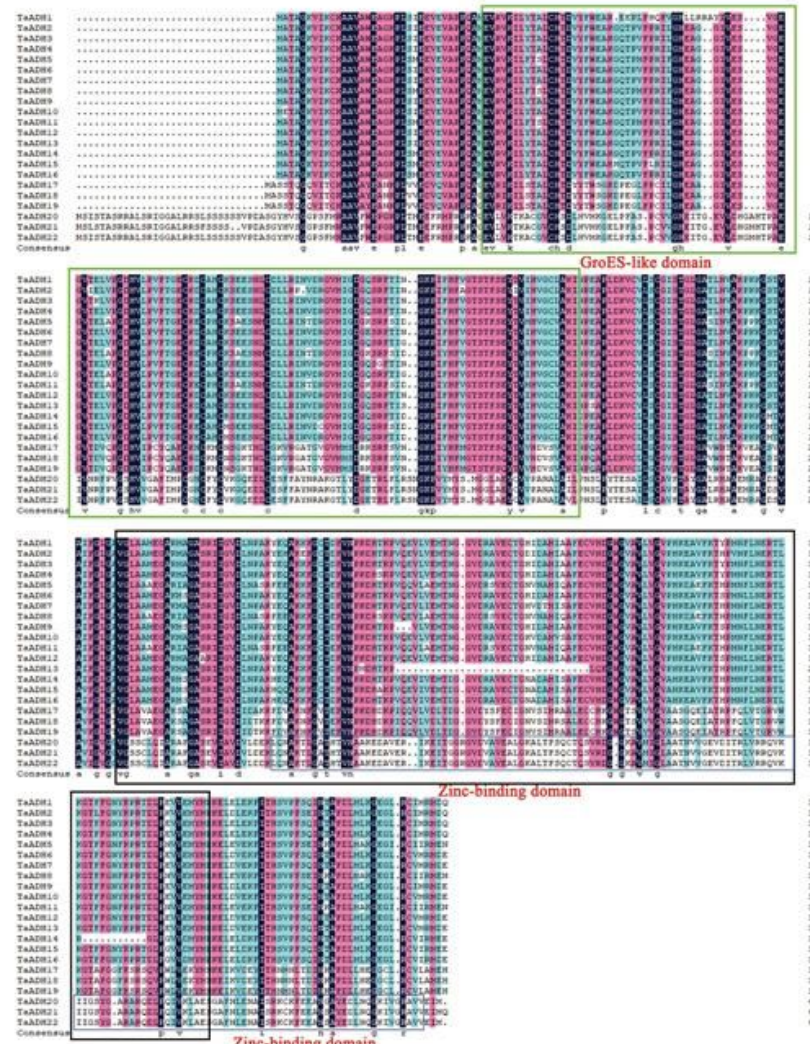

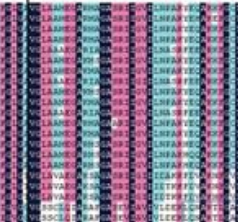

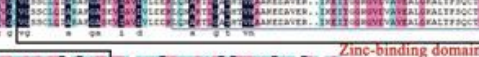
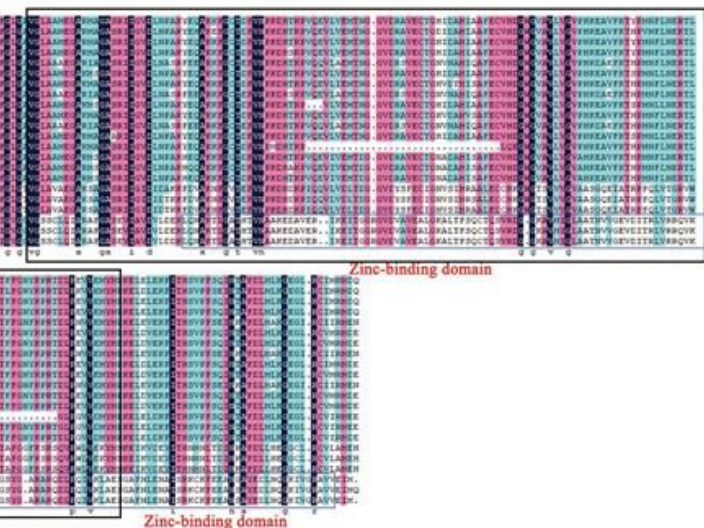

B
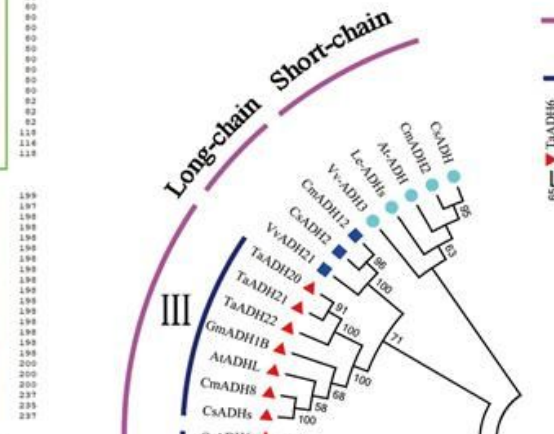

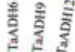
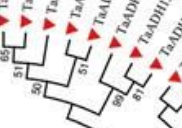

(1)

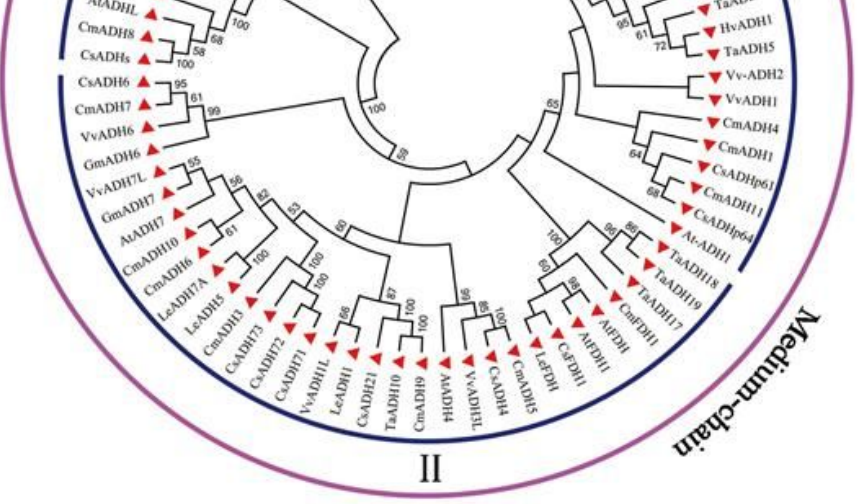

Figure 1

Sequences alignment and phylogenetic tree of TaADH proteins in wheat with other species. A. amino acid sequence alignment among TaADHs. Sequences were aligned using the DNAMAN5.0 Program. GroES-like domain and a structural zinc-binding domain were identified. B. phylogenetic tree among TaADHs and other ADHs. At: Arabidopsis thaliana, Cm: Cucumis melo, Cs: Cucumis sativus, Hv: Hordeum vulgare, Os: Oryza sativa, Gm: Glycine max, Le: Lycopersicon esculentum, Ta: Triticum aestivum, Vv: Vitis vinifera. 

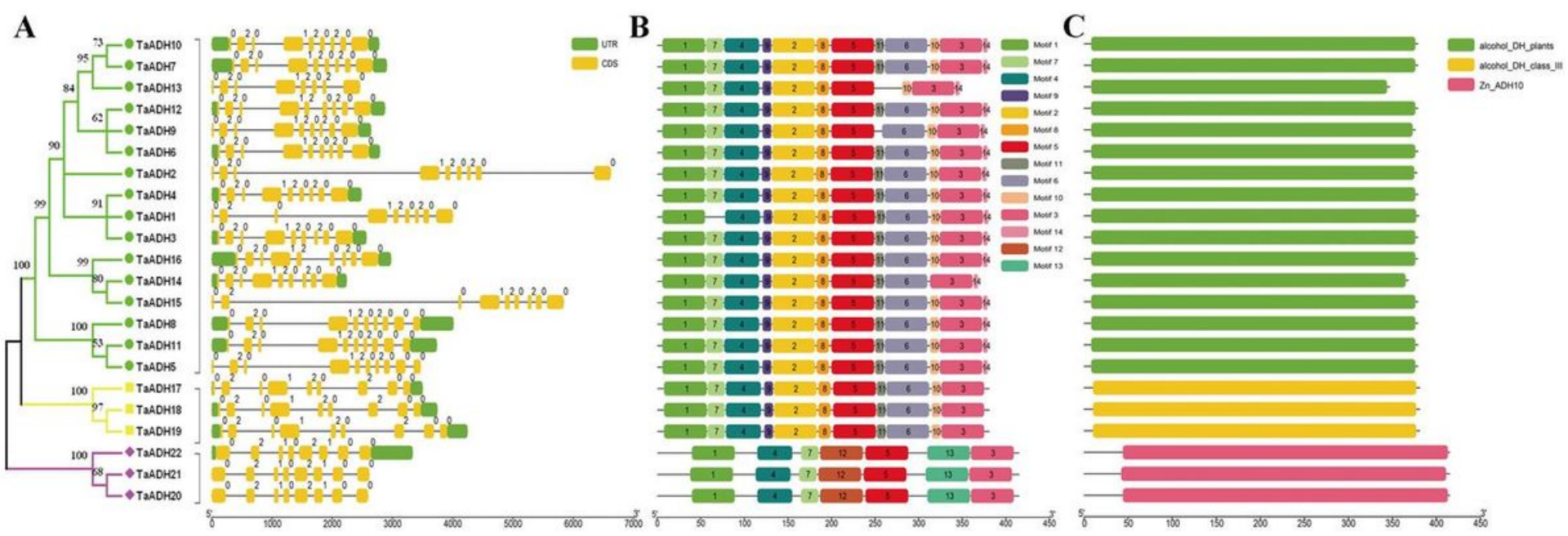

Figure 2

Exon-intron structures and motif compositions of TaADH proteins in wheat. The sequence information for each motif is provided in Fig. S1.

A

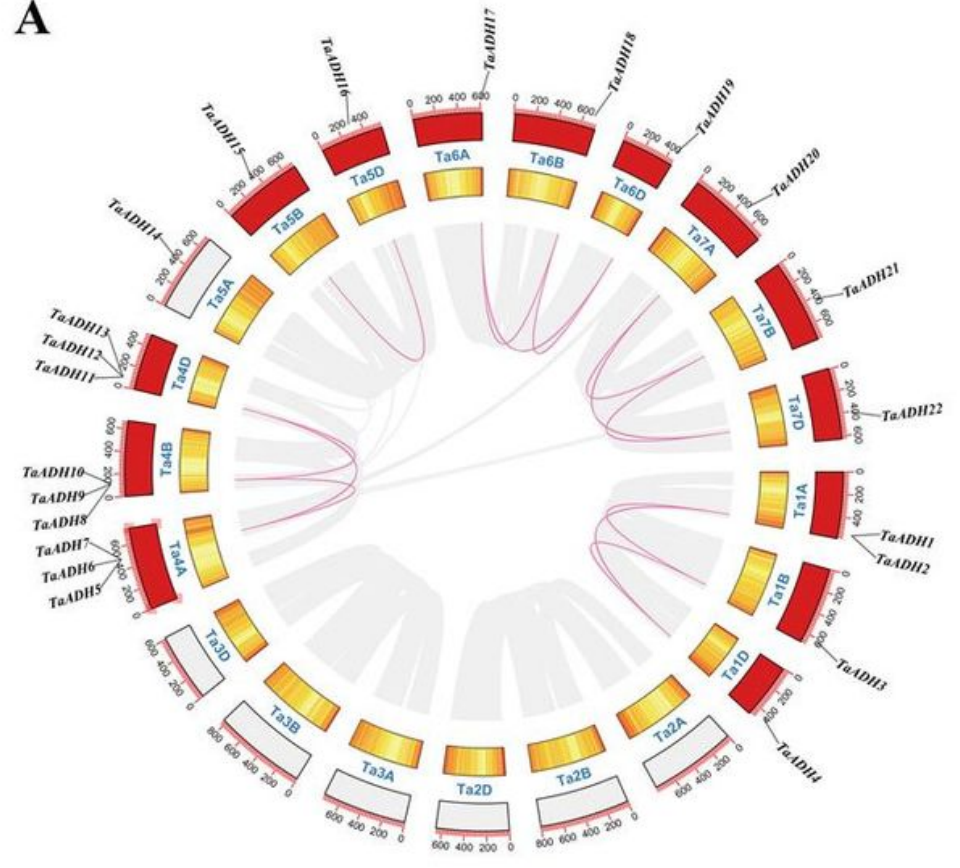

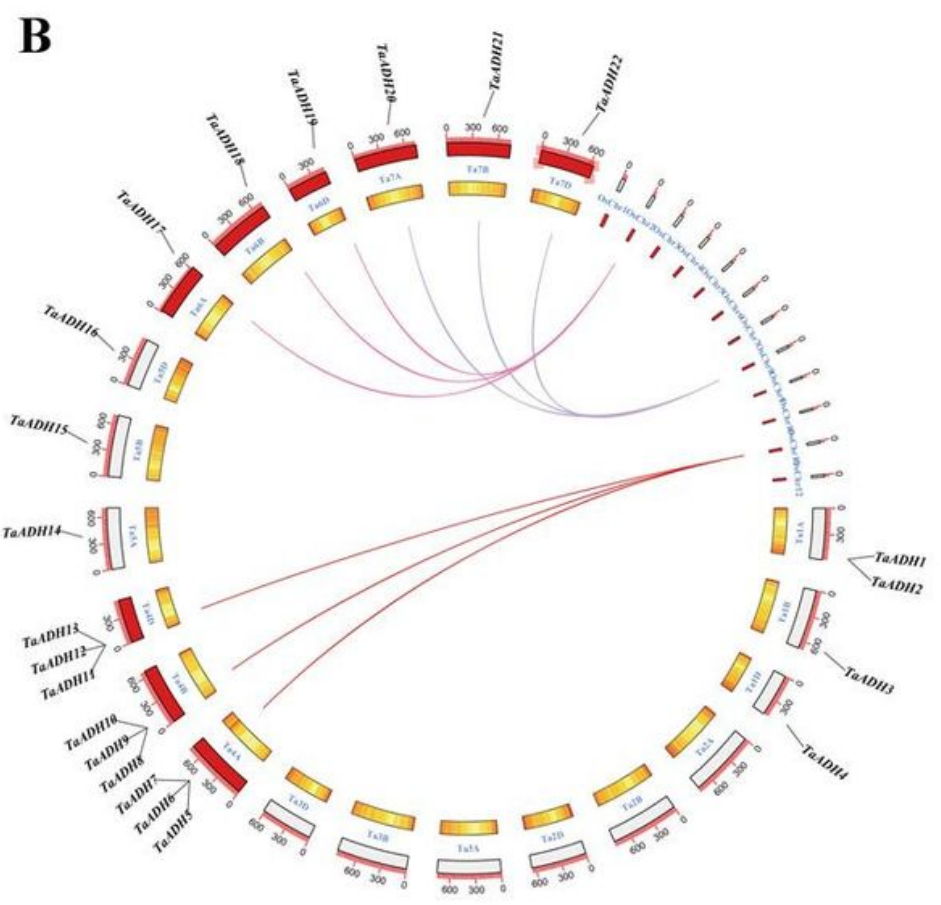

\section{Figure 3}

Chromosomes distribution and synteny analysis of TaADH genes in wheat. A. Ta1-7 represented chromosomes in A, B and D sub-genomes in Triticum aestivum. Red lines linked the syntenic orthologous in T. aestivum genomes. B. OsChr1-12 represented chromosomes in Oryza sativa. Red, pink and purple lines linked the syntenic paralogs within genomes of $\mathrm{T}$. aestivum and Oryza sativa. 
A

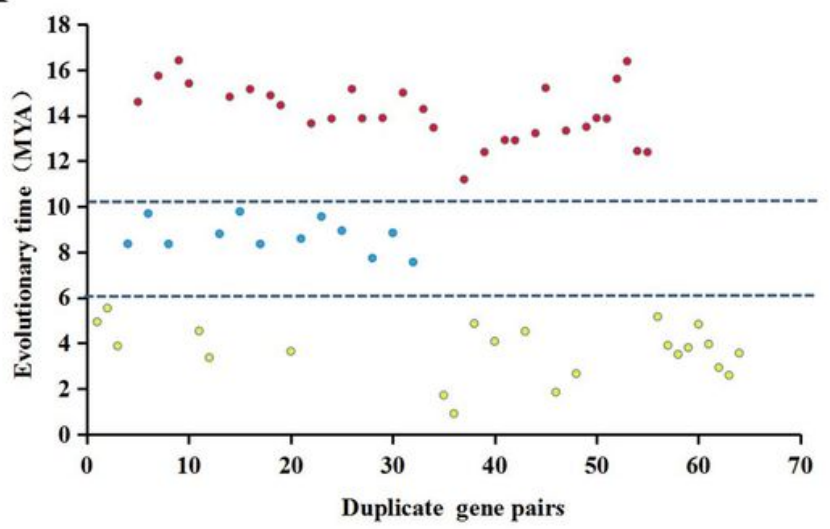

B

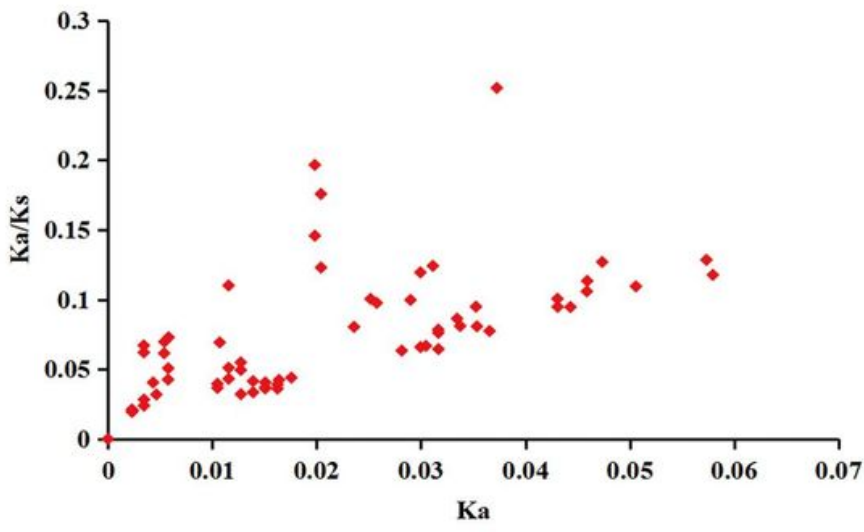

Figure 4

Evolutionary time and $\mathrm{Ka} / \mathrm{Ks}$ analysis of TaADH genes in the wheat genome. A. Evolutionary time for duplicated TaADH gene pairs; $\mathrm{B}$. Ka/Ks values for duplicated TaADH gene pairs

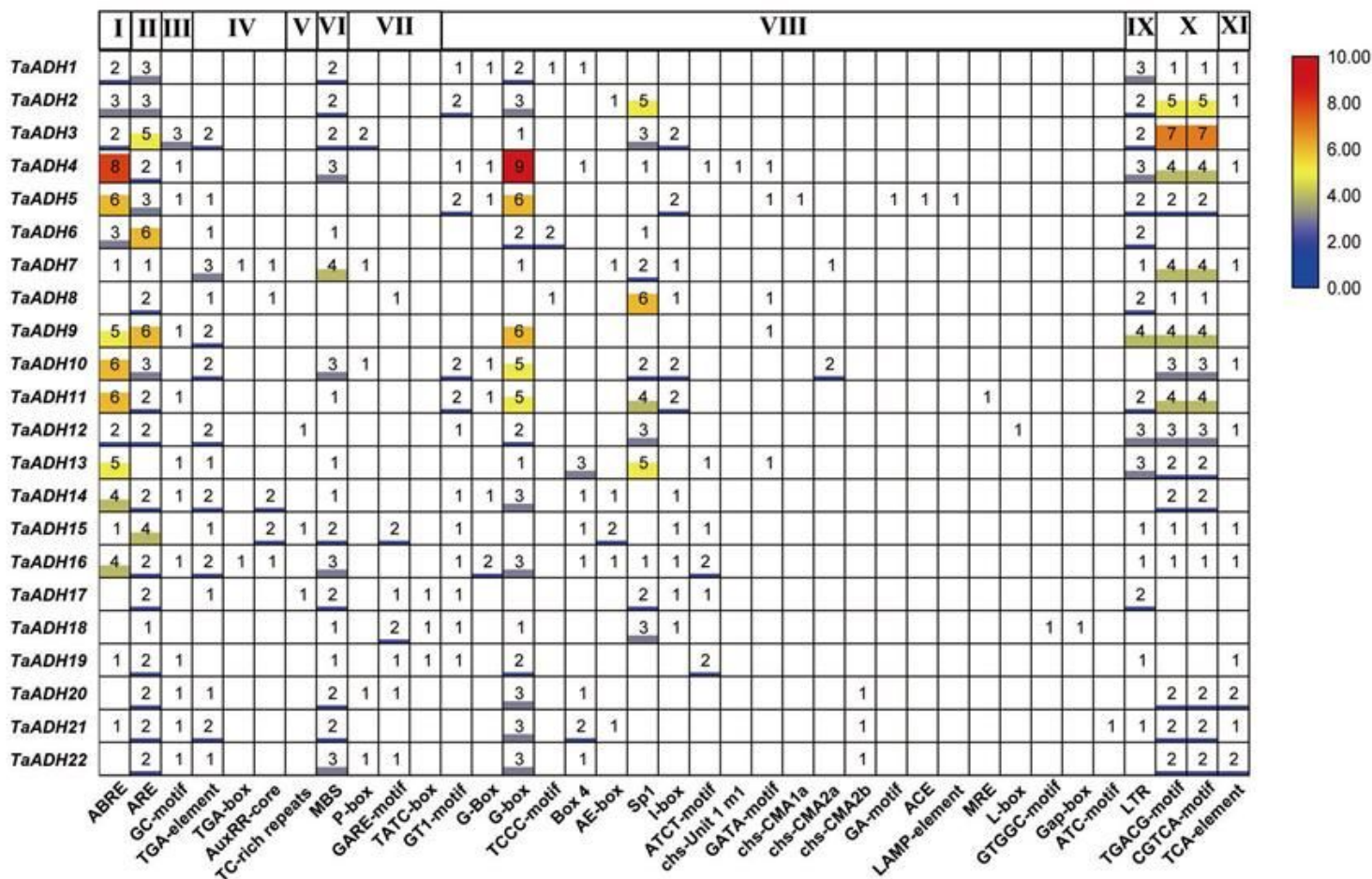

I Abscisic acid responsiveness II Anaerobic induction III Anoxic specific inducibility IV Auxin responsiveness

V Defense and stress responsivenes VI Drought-inducibility VII Gibberellin responsiveness VIII Light responsiveness

IXLow-temperature responsiveness $\quad$ X MeJA-responsiveness IX Salicylic acid responsiveness

\section{Figure 5}

Cis-acting elements distribution and statistical analysis of TaADHs promoters. The color of the square depicts the quantity of the predicted cis-acting elements in the promoter region. 


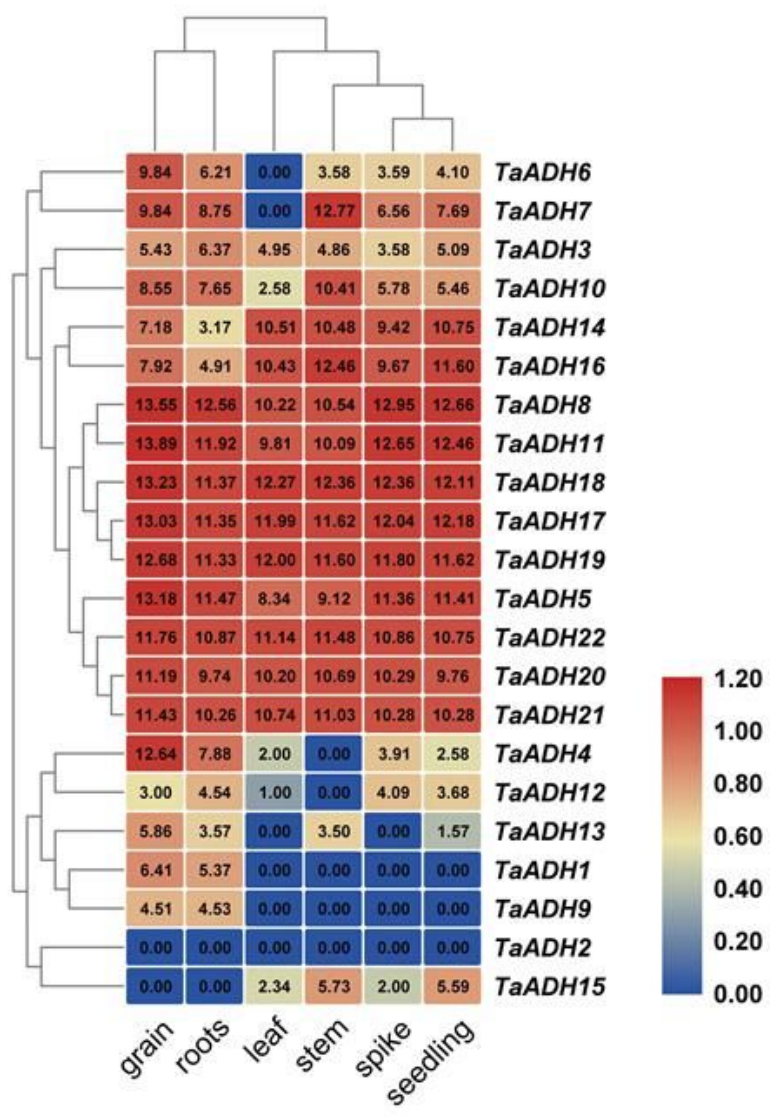

\section{Figure 6}

The expression of TaADH genes in wheat various tissues. A heatmap was created in TBtools software and based on the FPKM data (http://www.wheatexpression.com/) of different parts of Chinese spring wheat. The FPKM data was standardized on a base of 10. The color scale represents relative expression levels, with red indicating higher levels of expression and blue indicating lower expression levels. 


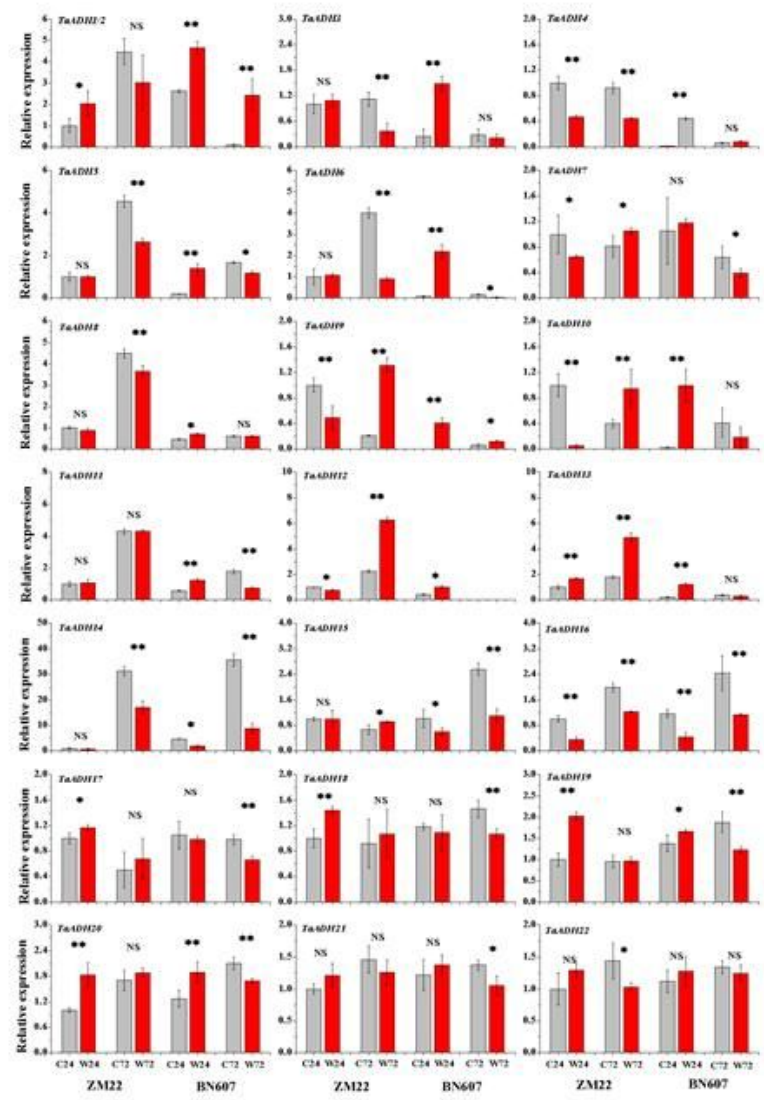

Figure 7

Expression profiles of TaADH genes in wheat seeds of waterlogging-tolerant and intolerant wheat varieties under waterlogging stress. ZM22: Zhoumai 22, BN607: Bainong 607; C24, after $24 \mathrm{~h}$ of germination under the control treatment; W24: after $24 \mathrm{~h}$ of germination under the waterlogging treatment; $\mathrm{C72}$, after $72 \mathrm{~h}$ of germination under the control treatment; W72, after $72 \mathrm{~h}$ of germination under the waterlogging treatment; Each point represents the average from three samples. The error bars represent the SDs. $*$ indicates significance at $p<0.05, * *$ indicates significance at $p<0.01, N S$ indicates No significance.

\section{Supplementary Files}

This is a list of supplementary files associated with this preprint. Click to download.

- Fig.S1.pdf

- Tables3.xlsx

- Tables2.xIsx

- Tables1.xIsx 